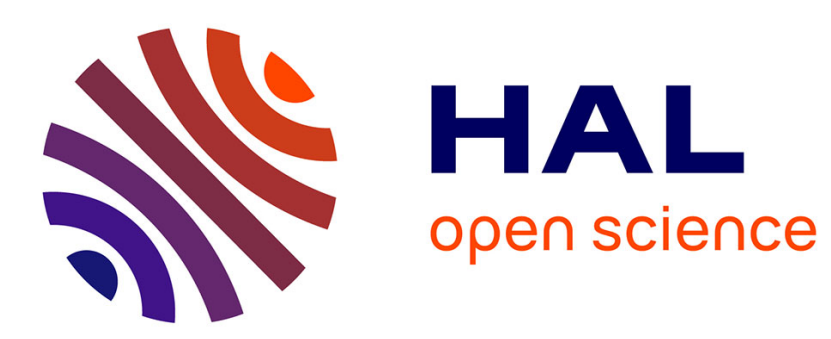

\title{
Fast Tracking of Fluid Invasion Using Time-Resolved Neutron Tomography
}

\author{
Clément Jailin, Maddi Etxegarai, E. Tudisco, S. A Hall, S. Roux
}

\section{To cite this version:}

Clément Jailin, Maddi Etxegarai, E. Tudisco, S. A Hall, S. Roux. Fast Tracking of Fluid Invasion Using Time-Resolved Neutron Tomography. Transport in Porous Media, 2018, 10.1007/s11242-018-1055-9 . hal-01800787

\section{HAL Id: hal-01800787 https://hal.science/hal-01800787}

Submitted on 28 May 2018

HAL is a multi-disciplinary open access archive for the deposit and dissemination of scientific research documents, whether they are published or not. The documents may come from teaching and research institutions in France or abroad, or from public or private research centers.
L'archive ouverte pluridisciplinaire HAL, est destinée au dépôt et à la diffusion de documents scientifiques de niveau recherche, publiés ou non, émanant des établissements d'enseignement et de recherche français ou étrangers, des laboratoires publics ou privés. 


\title{
Fast tracking of fluid invasion using time-resolved neutron tomography
}

\author{
C. Jailin · M. Etxegarai · E. Tudisco · S. \\ A. Hall • S. Roux
}

Received: date / Accepted: date

\begin{abstract}
Water flow in a sandstone sample is studied during an experiment in-situ in a neutron tomography set-up. In this paper, a projection-based methodology for fast tracking of the imbibition front in 3D is presented. The procedure exploits each individual neutron 2D radiograph, instead of the tomographicreconstructed images, to identify the 4D (space and time) saturation field, offering a much higher time resolution than more standard reconstruction based methods. Based on strong space and time regularizations of the fluid flow, with an a priori defined space and time shape functions, the front shape is identified at each projection time step. This procedure aiming at a fast tracking the fluid advance is explored through two examples. The first one shows that the fluid motion that occurs during one single $180^{\circ}$ scan can be resolved at $5 \mathrm{~Hz}$ with a sub-pixel accuracy whereas it cannot be unraveled with plain tomographic reconstruction. The second example is composed of 42 radiographs acquired all along a complete fluid invasion in the sample. This experiment uses the very same approach with the additional difficulty of large fluid displacement in between two projections. As compared to the classical approach based on full reconstructions at each invasion stage, the proposed methodology in the studied examples is roughly 300 times faster offering an enhanced time resolution.
\end{abstract}

Keywords Pressure-driven flow, Neutron tomography, 4D in-situ measurement, Model-driven inverse problem, Proper Generalized Decomposition

C. Jailin and S. Roux

LMT (ENS Paris-Saclay/CNRS/Univ. Paris-Saclay), 61 avenue du Président Wilson, F-94235 Cachan, France

E-mail: clement.jailin@ens-paris-saclay.fr

M. Etxegarai

Univ. Grenoble Alpes, CNRS, Grenoble INP,Institute of Engineering, Univ. Grenoble Alpes, 3SR, F-38000 Grenoble, France

E. Tudisco

Division of Geotechnical Engineering, Lund University, Lund, Sweden

S. A. Hall

Division of Solid Mechanics, Lund University, Lund, Sweden 


\section{Introduction}

Studies of fluid flow and transport in porous rock have received increasing interest related to a number of different resource engineering challenges, e.g., hydrocarbon production and $\mathrm{CO}_{2}$ sequestration, amongst other applications. Standard laboratory approaches to study such phenomena are based on "global" flow evolution (e.g., total sample saturation vs. time), which only provide spatial average measurements over the whole specimen. Local measurements of the saturation field during a fluid invasion process would however be highly desirable to validate and calibrate pressure driven flow and imbibition models, especially in samples where a heterogeneous microstructure affects porosity, permeability and wetting properties in an intimately coupled way, meaning that it is difficult to disentangle the influence of each one. Heterogeneity is particularly important, for example, in stratified media or mechanically deformed samples that may contain cracks or shear bands. In such cases, the fluid flow process is intrinsically a $4 \mathrm{D}$ phenomenon that requires dedicated $4 \mathrm{D}$ measurement methods [1,2]. Being able to image this fast time dependent fluid advance at high speed as in real cases is a challenge in the direction of the study of pressure driven flow.

The recent developments of fast synchrotron tomography [3] allow one to image $3 \mathrm{D}$ evolutions of fluid invasion inside samples at a fast rate $(20 \mathrm{~Hz}$ in [4,5]). Both X-ray [6,7,8,9] and neutron [10,11,12, imaging are used to image absorbing fluid (e.g., zinc iodide for X-rays [13,14,5] or water for neutrons). These two tomographic modalities can even be combined to benefit from their different contrast sensitivities as shown by Hall [15.

Many methods have been developed to extract the position of the fluid from 3D volumes or slices. From simple segmentation of the gray level images to more sophisticated method based on model driven evolution such as history matching [16. 13.17. History matching is an iterative inverse problem, commonly employed in petroleum reservoir engineering, whereby a reservoir simulation model is tuned to be consistent with experiment and accurately describes the fluid advance. However, since these identification methods are based on 3D volumes, they require quasistatic flows (although this constraint becomes less stringent with the progress towards increasingly fast 3D image acquisition). Fluid flow during a tomographic scan leads to blurring in the reconstructed volume around the fluid-front. Eventually, some acquired scans can not be reconstructed because of the discrepancy between the initial and final saturation states over the scan duration. One way to circumvent such difficulties is to resort to samples prepared for being invariant in one direction, and where the fluid advance is expected to be read easily in the radiographs [18, 19,20]. Armstronget al. proposed in [21] a method based on radiograph differences to extract the fluid advance with a higher time resolution.

Similar temporal issues to those described above can be found during mechanical testing in-situ with tomography where the sample may evolve (due to viscous effects, creep, relaxation, thermal variations, etc. ) during the tomographic acquisition. These effects lead to blurring that affects quantitative measurements based on the image contrast (i.e., full-field space-time measurement [22]). To address this problem, recent methods called Projection-based Digital Volume Correlation (P-DVC) [23, 24] and Discrete Digital Projections Correlation [25] have been developed for the measurement of 3D displacement fields based on radiographs rather than volumes. The P-DVC method, after a first (classical) tomography performed 
before the mechanical test, does not require more than two orthogonal radiographs per loading step of the mechanical test to identify the full 3D displacement field. This method, enriched with a model-based regularization, and a model reduction strategy to extract principal modes, makes fast-rate 4D imaging - even in laboratory tomographs — possible [26].

The principle of the P-DVC method is, at first order (i.e., unless iterative methods are used), the correction of a reference $3 \mathrm{D}$ volume by the weighted gradient of this volume so that its projections match with radiographs of the deformed volume. The method proposed in this paper, being a projection matching optimization, is in a similar vein. The final shape of a fluid volume is obtained from an acquisition of the saturated sample. This volume is then corrected iteratively to match, in the projected space, with radiographs at all invasion states. This optimization method is based on the minimization of the quadratic difference between the projected model of the fluid column and the real fluid advance.

The resolution of space-time evolution can be carried out with a separation of space description and time evolution using Proper Generalized Decomposition (PGD) techniques [27,28]. This method consists of successive enrichments of a field adding a new space-time direction at each iteration, each term of the sum being sought a priori in separate representations in space and time. This approach captures the most important modes first and allows one to stop when enough fine details have been incorporated. The space-time decomposition for measurement has been studied with PGD-DIC and PGD-DVC developed in [29,30] with one dimensional space functions.

The aim of this study is to capture the $4 \mathrm{D}$ evolution of fluid invasion (4D space-time evolution of the saturation front) at each neutron radiography time, that is at a rate of $5 \mathrm{fps}$, for the particular example of the study. First, a description of the method based on a space-time separation is proposed. This is followed by applications of the method to two test cases, which are extracted from an experiment that was initially designed for serial 3D measurements. This experiment involved imaging of water flow into a sandstone specimen by neutron tomography. Because the studied porous sandstone is reasonably homogeneous at the scale of the observations, the fluid flow can be pictured as a rather steep variation from a fully saturated lower part of the sample to a dry upper part. Hence, the fluid (or saturation) front is modeled as a moving 2D surface about which a saturation gradient may be added as a further refinement. The first test aims at tracking the flow at the radiography rate, i.e., $5 \mathrm{~Hz}$, during a single $3 \mathrm{D}$ scan, while the sample was being rotated. In this case, the front traveled by a short distance of about 8 voxels. This evolution cannot be resolved by a classical reconstruction procedure with the assumption that the front remains perfectly still during the full scan duration. The second test case addresses the entire invasion process, where the front sweeps through the entire sample height. The saturation profile and velocity field are described and compared with a front detection method from 3D reconstructed volumes. 


\section{Method}

\subsection{Projection based method}

Tomography, be it obtained with X-rays or neutrons, consists of recording (twodimensional) radiographs $\rho(\boldsymbol{r}, t)$ where $\rho$ is the intensity, $\boldsymbol{r}$ the position in the detector, acquired at the rotation angle $\beta(t)$ of the sample about the $z$ axis at the time of capture $t$.

To reconstruct a $3 \mathrm{D}$ volume from the radiographs, it is necessary to use a socalled "flat field", $\rho_{0}(\boldsymbol{r})$, that corresponds to the beam intensity at the same position in the detector. According to standard reconstruction procedure, the ratio $\rho / \rho_{0}$ is the dimensionless beam attenuation, whose logarithm $p(\boldsymbol{r}, t)=\log (\rho(\boldsymbol{r}, t))-$ $\log \left(\rho_{0}(\boldsymbol{r})\right)$ is the line sum of local absorption coefficient along the ray hitting the detector at position $\boldsymbol{r}$. In the following $p$ is called a "projection". In the present case of neutron tomography, the beam is approximated as being parallel and the volume $A(\boldsymbol{x})$, (where $\boldsymbol{x}=(x, y, z)$ designates the 3D coordinates) can be obtained from an inverse Radon transform of the projections, $p(\boldsymbol{r}, t)$.

For different reasons, the simple Beer-Lambert law used above to relate the length of neutron ray through the material to the log of the attenuation may be corrected by non-linear (so called "beam hardening", BH) corrections 31. This can be seen from fully homogeneously saturated or dried sections of the sample that are not reconstructed with a homogeneous gray level. This effect can however be exploited to compute the projection correction. A first reconstruction and segmentation of the volume allows this solid and fluid length to be measured. At this stage, the collected data should allow for the determination of the intensity reduction after a length traveled through the pure solid phase and in the mixed dry-fluid phase. It can be noticed that the dry part has a very low absorption and does not exhibit significant beam hardening effects compare with the fluidfilled part. Hence an intensity correction of the high absorption would correct the beam hardening of the fluid part without changing the dry part. A simple beam hardening correction proposed by Herman et al. 32. where the linear projection is used as part of a higher order polynomial projection has been used in this work. The correction of the projections as a function of a 5th order polynomial allows the beam hardening of the fluid-invaded part to be removed without changing the intensity of the dry part.

The proposed approach for the identification of the fluid advance in a porous sample is inspired by the Projection-based Digital Volume Correlation (P-DVC) technique [23]. It is important to note that the challenge here, in the fluid-tracking, is to achieve a 3D description of the advancing fluid front at the same temporal rate as the $2 \mathrm{D}$ projection acquisition, in contrast to the usual practice of performing a $3 \mathrm{D}$ reconstruction assuming a still state during the acquisition.

The starting point of the method is a reference ("standard") tomography scan, performed at the beginning of the experiment and, ideally, with a dry sample. This reference is used to extract a 3D image composed only of the shape of the projected fluid (as in 20]). (Note however that the initial state in the experiment presented later was not perfectly dry and a small layer of fluid was present at the bottom.) The aim is thus to determine a description of a scalar saturation field $S(\boldsymbol{x}, t)$ in $3 \mathrm{D}$ space. 
Introducing the porosity $\phi(\boldsymbol{x})$ of the porous medium, the projection can be written

$$
p(\boldsymbol{r}, t)-p_{0}(\boldsymbol{r}, t)=\Pi_{\beta(t)}[\phi(\boldsymbol{x}) S(\boldsymbol{x}, t)]
$$

where $\Pi_{\beta(t)}$ is the projection operator at angle $\beta(t)$.

This decomposition is illustrated in figure 1 . The rotation axis of the sample, denoted as $z$, is perpendicular to the neutron beam, and is one principal axis of the detector. A gray level color coding has been chosen for radiographs and a diverging color palette (where 0 is encoded as white, while positive and negative values have a different dominant color) is used for all residual images to facilitate reading.

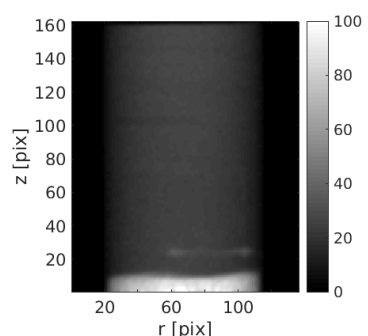

(a)

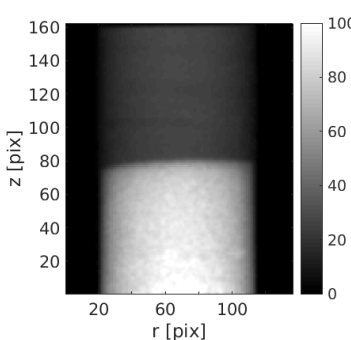

(b)

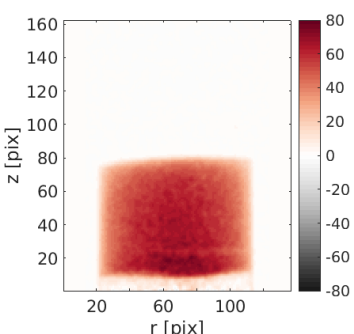

(c)

Fig. 1 Projections for (a) the reference state $p_{0}(\boldsymbol{r}, t),(\mathrm{b})$ a state during the invasion process $p(\boldsymbol{r}, t)$ and (c) the difference that shows the projection of the $3 \mathrm{D}$ column of fluid.

Based on the above description of the projections for a given saturation state, the determination of the $4 \mathrm{D}$ saturation field is performed through the minimization of a cost function that is chosen as the quadratic norm, $\chi^{2}$, of the residuals between the predicted and the actual projection data

$$
\eta(\boldsymbol{r}, t)=p(\boldsymbol{r}, t)-p_{0}(\boldsymbol{r}, t)-\Pi_{\beta(t)}[\phi(\boldsymbol{x}) S(\boldsymbol{x}, t)]
$$

thus

$$
\chi^{2}=\sum_{\boldsymbol{r}, t} \eta(\boldsymbol{r}, t)^{2}
$$

The minimized quantity is the norm of the residual field that gives a precious information on the quality of the solution because it highlights all patterns that have not been correctly captured (because of noise, artifacts, model error, illconvergence, etc. ) and it is based on the raw acquired data, with a minimal influence of prior assumptions that could bias the judgment.

\subsection{Fluid field model and parametrization}

Different descriptions of fluid flow in porous materials can be found in the literature, from detailed models accounting for presence or not of the fluid in the pore space, or more homogenized versions where the fraction of the pore space invaded by the fluid, i.e., the saturation $S$, is used. The choice of the description used is essential, as it enables tuning of the number of needed parameters, which 
has a drastic impact on our ability to invert the problem. The ideal choice is to restrict the saturation description to the smallest possible number of parameters yet maintaining a sufficient representation of the reality.

To have an accurate model for the projections, it is necessary to know the amount of fluid intersected by the neutron beam, which is given by the product of the saturation $S(\boldsymbol{x}, t)$ and the (connected) porosity $\phi(\boldsymbol{x})$ (independent of time).

The saturation itself can be determined by exploiting the following assumptions:

- First, the fluid "front" is assumed to be an abrupt transition in the saturation field where it drops to 0 in one pixel distance. It can be described by a single valued surface, $z=h(x, y, t)$, and hence $S=0$ for $z>h(x, y, t)$. This front moves upward as invasion takes place

- Second, behind the front, $z<h(x, y, t)$, saturation increases progressively in time and finally reaches a full saturation. The saturation profile seems to be well described by a function of the distance to the front $h(x, y, t)-z$, this property is not a universal property of pressure-driven flow, but it is observed to be well obeyed in the studied case.

The surface of the fluid front can be expressed, with a separation of variables, as a sum of modes, composed by the products of a spatial field and a temporal function, respectively $F^{m}$ and $\tau^{m}$

$$
h(x, y, t)=\sum_{m=1}^{N_{m}} \tau^{m}(t) F^{m}(x, y) .
$$

From this general expression of the front, (that is not restrictive) the spatial field can be specialized to a reduced space generated by $N_{s}$ shape functions, $\varphi_{i}(x, y)$ with $i=1, \ldots, N_{s}$, weighted by amplitudes $f_{i}^{m}$. Different levels of description are possible to regularize the space front. One is "free-form" from a fine finite-element mesh description of the surface. This description requires many degrees of freedom. A local "smoothing" can be added to constrain all independent nodal degrees of freedom, and obtain a better conditioned problem. Alternatively, a "strong" regularization can be defined extracting the saturation parametrization from a pressure driven flow model. The latter is generically the best choice, as it leads to very few degrees of freedom, although it does require a physically-relevant model of the saturation consistent with the scale of observation.

It is desirable to reduce the number of degrees of freedom, to couple them together for better conditioning and to ease dialogue with models. The fluid front can thus be written as

$$
h(x, y, t)=\sum_{m=1}^{N_{m}} \tau^{m}(t) \sum_{i=1}^{N_{s}} f_{i}^{m} \varphi_{i}(x, y) .
$$

The space shape functions, $f_{i}^{m}$, can be tuned to adjust their spatial resolution: from voxels (Dirac) representation, to finite element shape function for a $2 \mathrm{D}$ mesh of the cross section, or to polynomials of low order, for example. In the same spirit, the time evolution can be decomposed over temporal shape functions, $\theta_{l}(t)$,

$$
h(x, y, t)=\sum_{m=1}^{N_{m}}\left(\sum_{l=1}^{N_{t}} g_{l}^{m} \theta_{l}(t)\right)\left(\sum_{i=1}^{N_{s}} f_{i}^{m} \varphi_{i}(x, y)\right) .
$$


It can be noted that only the norm of the product $\boldsymbol{g}^{m} \boldsymbol{f}^{m}$ matters so that an additional convention such as: $\max \left\|\sum_{i=1}^{N_{s}} f_{i}^{m} \varphi_{i}(x, y)\right\|=1$, has to be chosen without consequence.

\subsubsection{Parametrization of bulk saturation}

Detailed study of the images of the invasion process indicates that (at least in the regime relevant for our experiment) the saturation shows a rapid first increase from 0 to an intermediate value followed by a slower second stage increase up to 1. This secondary stage can be seen as being ruled by a unique 1D function of the vertical distance to the front. Following this observation, the saturation can be written as

$$
S(\boldsymbol{x}, t)=\zeta_{\boldsymbol{p}}(h(x, y, t)-z),
$$

where $\zeta_{\boldsymbol{p}}$ is a smooth function of its argument, null for negative values, providing the abrupt cut-off at the front. This function $\zeta_{\boldsymbol{p}}$ can be parametrized by a set of parameters, collectively denoted as a vector $\boldsymbol{p}$.

As a final step, to soften the very steep vertical variation occurring at the front, the above saturation field is convoluted with a Gaussian along the $z$ direction. An additional component in the vector $p$ will refer to the width of this Gaussian. Since $\zeta$ is a slowly varying function in $z$ except at the front where it is discontinuous, the effect of this last smoothing operation only affects the immediate vicinity of the front and has no perceptible influence on the bulk saturation.

\subsection{Inverse problem resolution}

The previous section described how the saturation field is described by the front (with parameters gathered in two vectors $\boldsymbol{f}$ and $\boldsymbol{g}$ ) and by the bulk saturation fields with parameters $\boldsymbol{p}$. In this section, the solution to the inverse problem is described.

Although the best was done to introduce the fewest possible number of unknown parameters to describe the fluid advance, their number is still too large for the problem to be tractable. The PGD framework [27,28] to progressive enrichment of the model description with modes is both a very powerful and convenient approach. At each iteration of the algorithm, the fluid front can be written as the sum of previously identified modes plus a new one. Hence,

$$
h^{(m)}(x, y, t)=h^{(m-1)}(x, y, t)+\left(\sum_{l=1}^{N_{t}} g_{l}^{m} \theta_{l}(t)\right)\left(\sum_{i=1}^{N_{s}} f_{i}^{m} \varphi_{i}(x, y)\right) .
$$

The minimization of the functional leads to the determination of the unknowns from 3 coupled equations,

$$
\begin{aligned}
\boldsymbol{f}^{m} & =\operatorname{Argmin}_{f}\left(\chi(\boldsymbol{f}, \boldsymbol{g}, \boldsymbol{p})^{2}\right), \\
\boldsymbol{g}^{m} & =\operatorname{Argmin}_{g}\left(\chi(\boldsymbol{f}, \boldsymbol{g}, \boldsymbol{p})^{2}\right), \\
\boldsymbol{p} & =\operatorname{Argmin}_{p}\left(\chi(\boldsymbol{f}, \boldsymbol{g}, \boldsymbol{p})^{2}\right) .
\end{aligned}
$$


To solve this coupled system, a fixed point procedure is used, solving for the above three minimization problems successively, keeping the other two sets of parameters fixed, as described in Algorithm 1. It was observed that a very low number $(3-5)$ of iterations was needed to reach a stable solution, and an ill-convergence escape condition based on a maximum number of iterations was never reached. Even if the two space and time unknown amplitudes are defined by the norm of their products, the fixed point algorithm, composed of separated equations leads to a correct determination (from the choice of the initialization of $\boldsymbol{g}^{m}$ from which $\boldsymbol{f}^{m}$ is computed) allowing convergence. The choice of a norm affecting each unknown can finally be set (or reset) at the end without consequence.

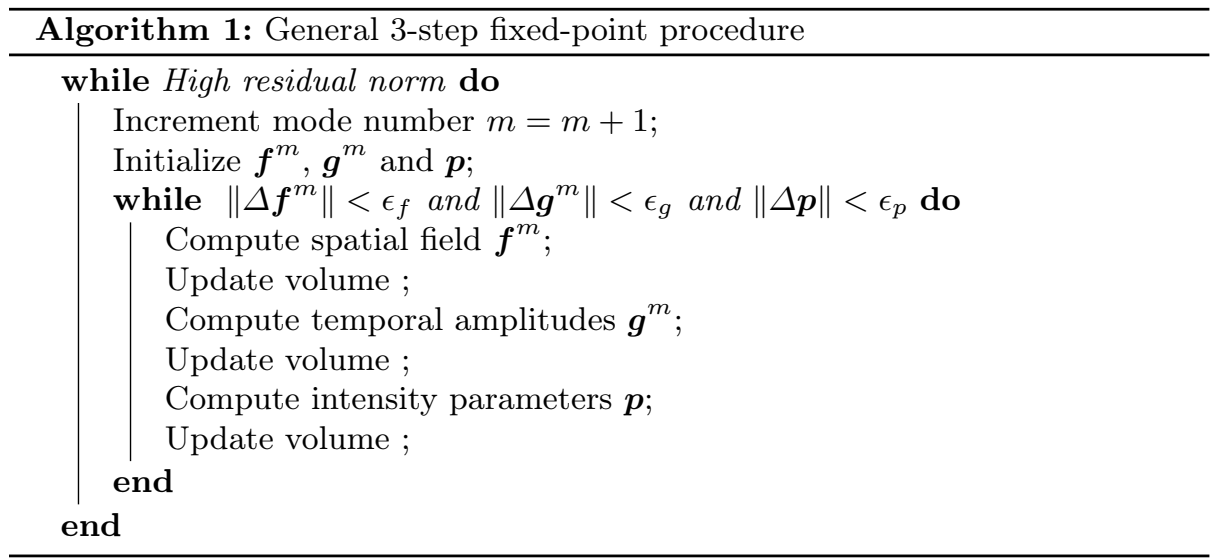

The three parts of the fixed points are obtained from the above functional using a Newton algorithm. The derivative with respect to $\boldsymbol{h}$ leads to

$$
\boldsymbol{f}=\boldsymbol{N}^{-1} \boldsymbol{n}
$$

with $\boldsymbol{N}$ the spatial Hessian matrix of $\chi^{2}$ with respect to $\boldsymbol{f}\left(i . e ., N_{i j}=\partial_{f_{i}} \partial_{f_{j}} \chi^{2}\right)$, built from the sensitivity field, $\Psi_{i}^{m}(\boldsymbol{r}, t)$, i.e., the variation of the projection for an infinitesimal variation of input parameter,

$$
\Psi_{i}^{m}(\boldsymbol{r}, t)=\frac{\partial \Pi_{\beta(t)}[\phi(\boldsymbol{x}) S(\boldsymbol{x}, t)]}{\partial f_{i}^{m}}
$$

$\boldsymbol{n}$ is the second member vector based on the residual field, which can be written

$$
\begin{aligned}
N_{i j} & =\sum_{t, \boldsymbol{r}} \Psi_{i}^{m}(\boldsymbol{r}, t) \Psi_{j}^{m}(\boldsymbol{r}, t), \\
n_{i} & =\sum_{t, \boldsymbol{r}} \eta(\boldsymbol{r}, t) \Psi_{i}^{m}(\boldsymbol{r}, t) .
\end{aligned}
$$

The exact same formulation is found for the derivative with respect to $\boldsymbol{g}$ and $\boldsymbol{p}$. It is noteworthy that $\boldsymbol{p}$ does not depend of $m$ in the fixed point procedure. It is hence an update of this parameter that is performed at each mode identification. 


\section{Applications}

\subsection{Presentation of the test}

The studied sample is made of a porous sandstone from the Vosges mountains in the east of France 33. The sample shape is cylindrical (diameter $38 \mathrm{~mm}$ and height $80 \mathrm{~mm}$ ) with two opposing flattened surfaces on which two shallow notches were cut for a related study on the formation of localized deformation under triaxial compression (see [15,34]). The sample geometry is shown in Figure 2(a).

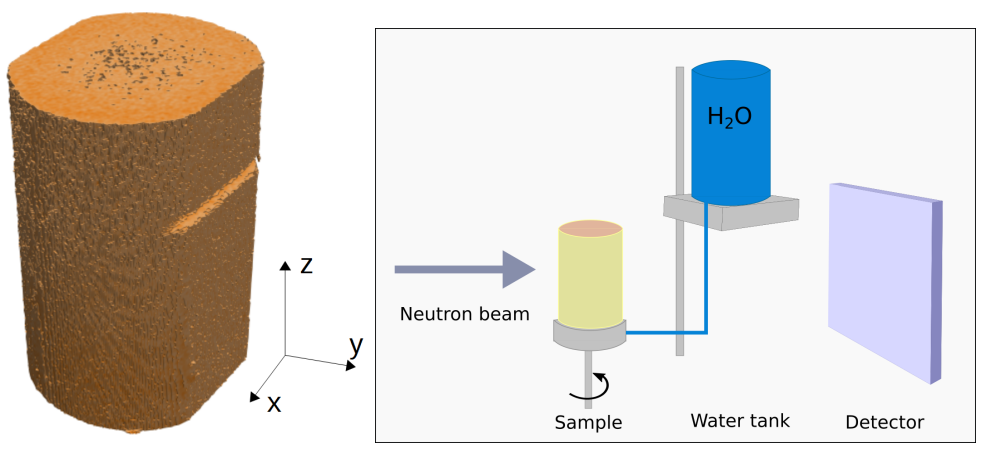

(a)

(b)

Fig. 2 (a) Cylindrical sandstone sample with two, opposing flattened surfaces each containing a notch, which are offset to each other. In the pressure-driven flow experiment, the fluid entered the sample from the bottom. (b) Scheme of the experimental setup. The water tank can be moved up and down to keep a constant pressure during the experiment

The fluid advance in the sample was imaged with neutron tomography at Helmholtz-Zentrum facility in Berlin (HZB) 35]. For the experiments, the sample was wrapped with thin PTFE tape then two Teflon inserts were placed on the flattened surfaces to make the overall shape cylindrical and a heat-shrink FEP membrane, which is reasonably transparent to neutrons, was shrunk to fit tightly around the sample and inserts. The PTFE tape was placed between the sample and the inserts to avoid preferential flow paths. The dry sample was placed and sealed with silicon in a cup that could be fed, by a tube from the bottom, with distilled water. The experimental setup, described in details in [36], is shown figure 2(b). Water was supplied to the cup at the base of the sample and the fluid advance was captured with 137 neutron tomography scans over a period of 2 hours.

The pressure of the water was given from the height of the water tank, which was adjusted during the experiment to compensate the water loss and keep the pressure constant. The fluid advance in the sample was driven more by the water pressure head than by capillarity.

Neutron tomographies were acquired with an acquisition time of 0.2 sec per radiograph and 307 projections over $180^{\circ}$, for a total scan time of $1 \mathrm{~min}$. The rotation of the table was continuous with a fixed velocity. 130 flat-fields and darkfields were acquired and used to perform a standard flat-field correction. The images are $541 \times 648$ pixels $^{2}$ with a pixel size of $110 \mu \mathrm{m}$. For a reduced computation 
time and because the front did not reveal any high spatial frequencies, the images have been down-sampled with a factor of 4 (i.e., after a Gaussian filtering of the images with a characteristic length of 4 voxel size, the smaller images are created sampling 1 pixel out of 4 in all space directions). Hence the images treated in the following are $138 \times 162$ pixels $^{2}$ with a pixel size of $440 \mu \mathrm{m}$.

To estimate the actual porosity field of the sample, $\phi(\boldsymbol{x})$, the final state after steady fluid invasion was reconstructed from a complete $180^{\circ}$ scan reconstruction using an algebraic SIRT procedure from the ASTRA Toolbox [37] similar to the initial state reconstruction. Because the first scan was performed with a small quantity of fluid in the bottom of the sample, a mask was applied to the bottom part of the projections. The following surface height is defined from $z_{0}=20$ voxels.

\subsection{Choice of parameterization}

\subsubsection{Spatial parameters}

The saturation front is described using a very simple model: a sum of low order bivariate polynomials of order 3, thus 10 degrees of freedom for the surface function are allowed for each mode.

\subsubsection{Temporal parameters}

A single projection cannot be used to identify all modes and indeterminacies would result if each single time step (and hence projection angle) were treated independently. This can be resolved by describing the time evolution by time shape functions - denoted as $\theta_{l}(t)$ in Eq. 6- that span several time steps or angles; these also introduce an additional smoothness. Again, the limit is that this smoothening should be consistent with reality and should not enforce an oversimplified definition of the front.

The time evolution is chosen to be expressed on a reduced basis composed of low order polynomials. Because the first case example has a simple and low frequency fluid advance, the time regularization was set using polynomials of order up to 2 . The second test case is more complex and was regularized in time with polynomials up to 4 th order. The proposed regularizations can be decided to be valid, or not, based on the residual field at the end of the identification procedure.

\subsubsection{Bulk saturation parameters}

The bulk saturation of the porous medium behind the invasion front $\sigma(\boldsymbol{x}, t, p)=$ $\zeta(h(\boldsymbol{x}, t)-z)$ is described with two parameters, $p_{1}$ and $p_{2}$, and an exponential function.

$$
\zeta(\delta h)= \begin{cases}0 & \delta h<0 \\ 1-\left(1-p_{1}\right) \exp \left(-\frac{\delta h}{p_{2}}\right) & \text { else }\end{cases}
$$

The saturation jump at the front is considered as a discontinuity from 0 to $p_{1}$. Although abrupt, the saturation evolution at the front is better described if the transition is smeared over a small distance by a convolution of $\zeta$ with a vertical 
Gaussian kernel of width 2 voxels. Note that this smoothing can be performed in the detector space for a faster computation.

The plot of the identified $\zeta(\delta h)$ at the end of the procedure can be used to validate the chosen intensity model (in the example, $p_{1}=0.64$ and $p_{2}=7.7$ vox); for the current example this is shown in Figure 3 . The chosen and identified profile, especially after the Gaussian smoothing are close to the profile extracted from the real data. Note that the reconstructed $3 \mathrm{D}$ volume may have been blurred because of fluid motion during the acquisition.

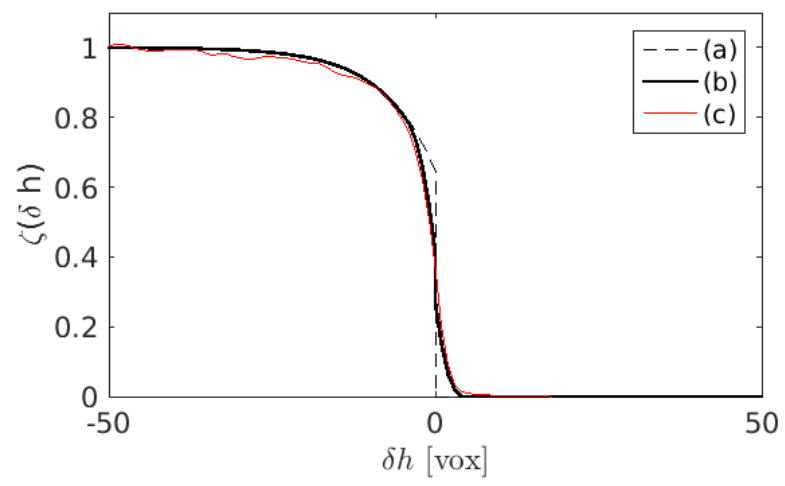

Fig. 3 Comparison of the mean vertical intensity lines, from the model $\zeta$ ((a) black dashed curve), the blurred model with a Gaussian of two vertical pixels ((b) black bold plain curve) and experimental data ((c) red thin curve) extracted from a $3 \mathrm{D}$ reconstruction.

\subsection{Case 1: during a scan rotation}

The first test case involves the measurement of the fluid advance from the radiographs acquired during a standard tomographic scan. The considered data were extracted from the middle of entire experiment and involve 307 radiographs that were acquired at $5 \mathrm{~Hz}$ frequency over 60 seconds during a continuous rotation of the sample from $\beta=0^{\circ}$ to $180^{\circ}$.

The projections of the fluid column for the first and last radiographs are shown in Figure 4 The difference between the first radiograph and the mirror symmetry of the last one indicate that the fluid front clearly moved during the $1 \mathrm{~min}$ acquisition. Such an evolution would blur the reconstructed 3D fluid field and, therefore, influence any subsequent 3D analysis.

The identification procedure consists of 3 modes, each composed of 2 unknowns for the saturation profile (vector $\boldsymbol{p}$ ), 10 unknowns in the spatial domain $f_{i}^{m}$ and 307 (resp. 3) unknowns in the time domain, $g_{l}^{m}$, for a free identification (resp. for a 2 nd order polynomial).

\subsubsection{Identification of the first mode for the fluid front}

The first spatial and time evolution is shown in figure 5. The range of variation of 


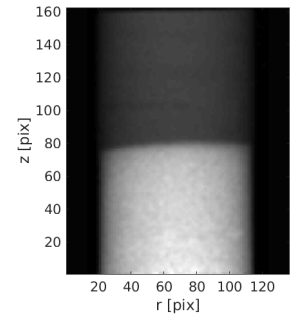

(a)

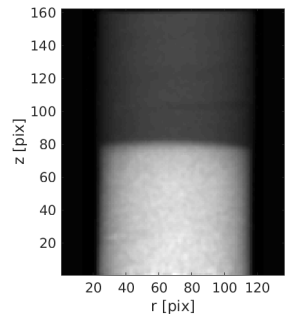

(b)

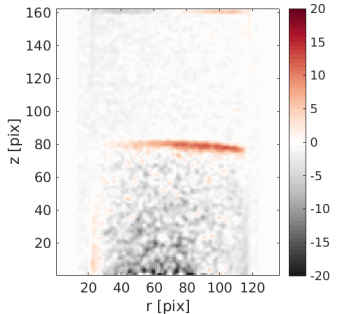

(c)

Fig. 4 Projection of the 3D column of fluid at the first (a) and last (b) angle. The difference between the first and the (mirrored) last image in (c) shows the fluid advance during the scan.

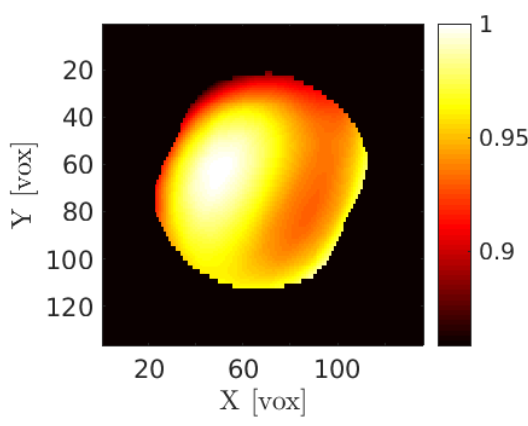

(a)

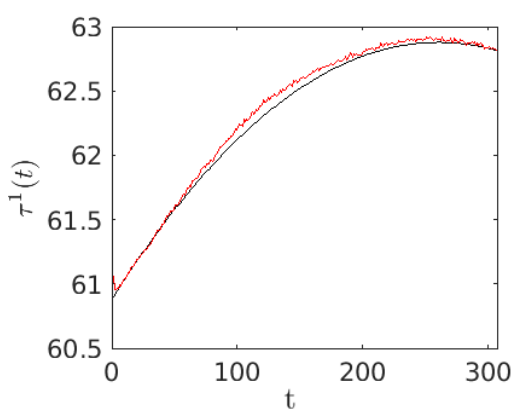

(b)

Fig. 5 Results of the first mode identification with (a) the space field of the surface and (b) the time evolution of the front $g^{1}(t)$ in black and the free identification in red.

the first spatial mode, about 0.1 , is small relative to its mean value (about 0.9), and hence the time evolution, to first order, reveals an invasion of the fluid into the sample that is initially fast and then more slowly saturates the region of the sample by the end of the $60 \mathrm{~s}$ acquisition. The variation of the mean front position is a displacement of approximately 2 voxels at scale 4 (hence 8 voxels at scale 1 , for a full resolution reconstruction). It is possible to extract the amplitude at each single time step by taking into account only this first spatial field and to use it to match projections. This provides a free-form estimate of amplitudes $f_{i}^{1}$ for local time shape functions, $f_{i}^{1}(t)=\delta\left(t-t_{i}\right)$. Such amplitudes are shown in red in Figure $5 \mathrm{~b}$. If instead, the time shape functions are chosen as polynomials of order 2 , the resulting determination of amplitudes is shown as the black curve. A fair agreement is observed, in spite of the drastic reduction in the number of degrees of freedom (from 307 down to 3). Moreover, this reduction comes with a much more robust (noise resilient) behavior. In addition to describing the mean volume invasion, the non-trivial front shape also captures a persistent non-uniformity of the front. However, higher mode orders are expected to contribute significantly to a finer description of the front shape.

The true metric to evaluate the merit of our procedure is the residual field $\eta(\boldsymbol{r}, t)$ which shows everything that has not been captured by the procedure (noise, 
artifact, model error etc. ). To evaluate the relevance of the non-trivial front shape of the first mode, it is easy to perform the same analysis with only one mode, but restricting the spatial part of $F$, to a single degree of freedom, namely a constant height (polynomial order equal to 0). An arbitrary choice of the amplitude of the space and time function has to be chosen. It was decided to set the maximum space amplitude to 1 to help for a visual understanding of the surface evolution. At convergence, the final residual, is shown in figure 6 at the beginning and end of the scan. The most critical part of this residual field that has to be scrutinized is the fluid surface zone (interface between empty and saturated pore volume). It can be seen that the mean evolution is well captured, but alternate positive and negative values show that this description is still inaccurate. The value of $\chi^{2}$ reaches 144 for a flat front fixed in time, and drops down to 103 when a variation in time is considered, showing that the latter choice allows one to improve significantly the solution. Let us note that with a single mode, when higher polynomial orders are considered in space, the residual decreases only slightly to 102 . When more modes are added, the spatial enrichment reveals much more rewarding as detailed in the following.

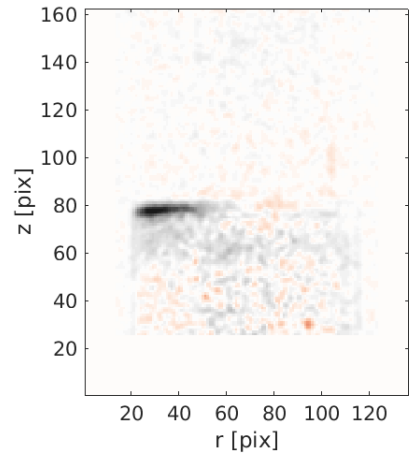

(a)

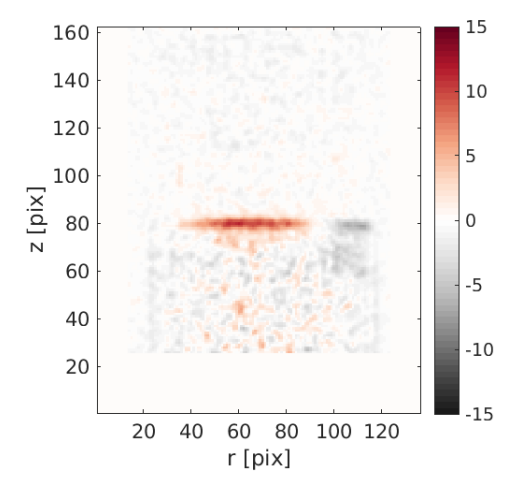

(b)

Fig. 6 Selected residual fields at (a) $\beta=0^{\circ}$ or $t=0 \mathrm{~s}$ and (b) $\beta=180^{\circ}$ or $t=60 \mathrm{~s}$ at convergence, with a flat and horizontal (constant height) surface.

\subsubsection{Identification of additional modes}

A comparison of the final residual field considering different time regularization is proposed in table 1 . From these results it can be seen that, a third order polynomial for the time evolution is required to correctly capture the fluid advance.

Most of the residuals are erased with 3 modes, which indicates that a solution for the front shape has been found. The final residual field is shown in figure 7 . Some structure can still be distinguished in the residuals below the front, which likely corresponds to reconstruction artifacts from the projected final fluid column.

The identification procedure was been stopped after the identification of 4 modes because the norm of the residual fields indicated convergence. The evolution 


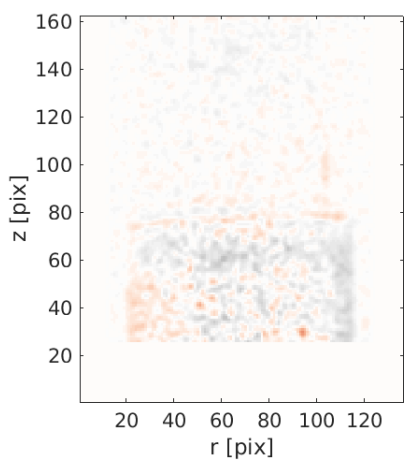

(a)

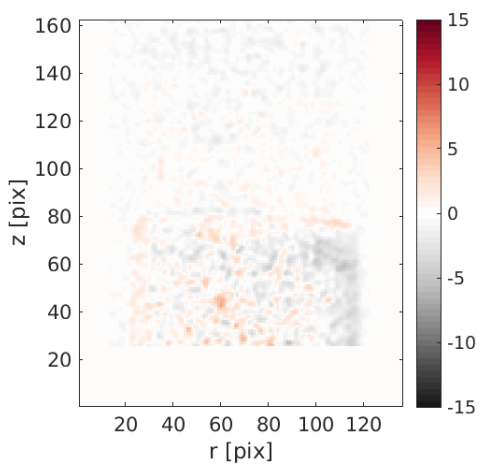

(b)

Fig. 7 Evolution of the selected residual fields, after correction with the three modes at (a) $\beta=0^{\circ}$ or $t=0 \mathrm{~s}$ and (b) $\beta=180^{\circ}$ or $t=60 \mathrm{~s}$ at the end of the procedure.

of the norm of the residual fields is presented table 1. It can be seen that the

Table 1 Evolution of the norm of the all residuals at convergence for different spatial fields polynomial orders and after successive mode identification

\begin{tabular}{|c|c|c|c|c|}
\hline \multirow{2}{*}{ Spatial basis order } & \multicolumn{4}{|c|}{ Number of modes } \\
\cline { 2 - 5 } & 1 & 2 & 3 & 4 \\
\hline 0 (flat) & 103 & 101 & 101 & 101 \\
1 & 102 & 84.1 & 83.7 & 83.5 \\
2 & 102 & 74.5 & 74.2 & 73.8 \\
3 & 102 & 69.4 & 68.7 & 68.5 \\
\hline
\end{tabular}

residual field does not decrease further after mode 3 , indicating that the main trends have been captured. The space time evolution (with a product expressed in voxels) of the different modes is shown in figure 8 .

The final surface can be reconstructed from the three identified modes. In figure 9, the front surface at two time steps are plotted. The evolution from time $t=0 \mathrm{~s}$ and $t=60 \mathrm{~s}$ is shown with the same color coding. It can be noted that the surface curvature changes progressively during invasion over the scan time.

\subsubsection{Comparison with $3 D$ front extraction method}

Using classical tomographic approaches, the beam hardening corrected projections can be used to reconstruct a 3D volume (assuming implicitly a steady fluid), from which a segmentation method, based on the value where the front saturation drops to 0 can be used to extract the shape and position of the saturation front. The measurement of the surface height can thus be determined. Obviously, this method allows a single front to be extracted for the time interval, which should be, in some way, an intermediate mean position. If the $3 \mathrm{D}$ front is determined by standard segmentation procedures, its height will be given with integer voxel resolution. Figure 10 shows the difference between the interface extracted from 


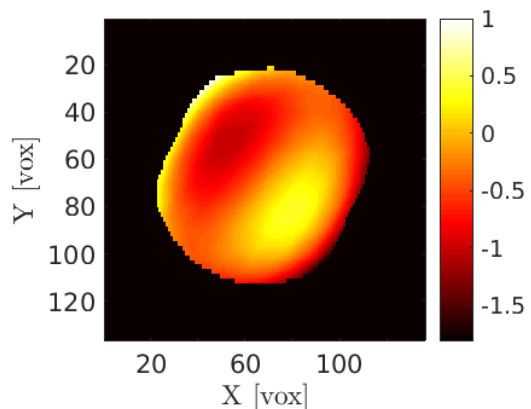

(a)

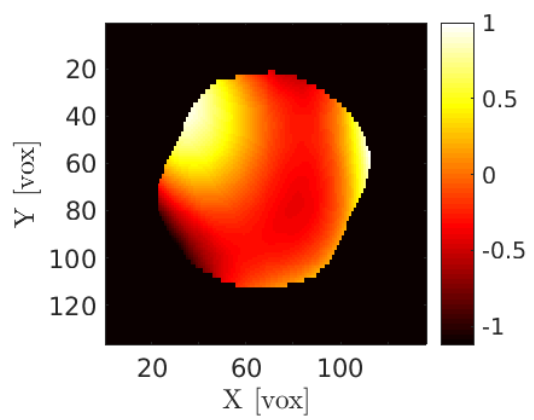

(c)

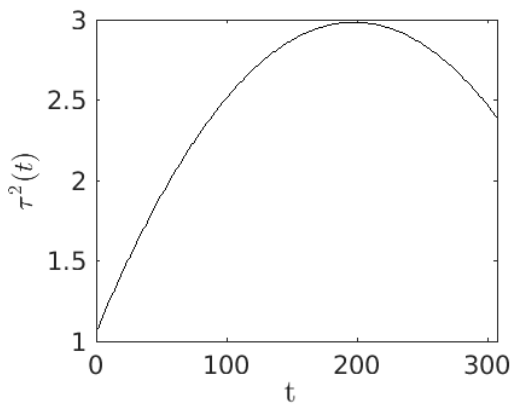

(b)

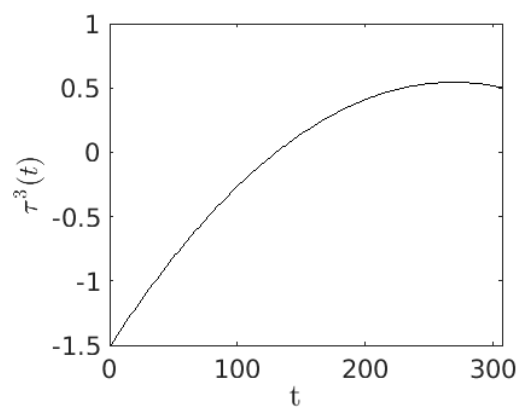

(d)

Fig. 8 Surface modes $m=2$ and 3. (a-c) is the space surface $F^{m}(x, y)$ and (b-d) is the time evolution $g^{m}(t)$

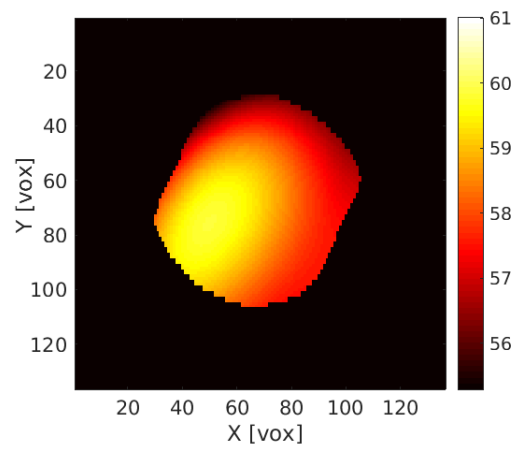

(a)

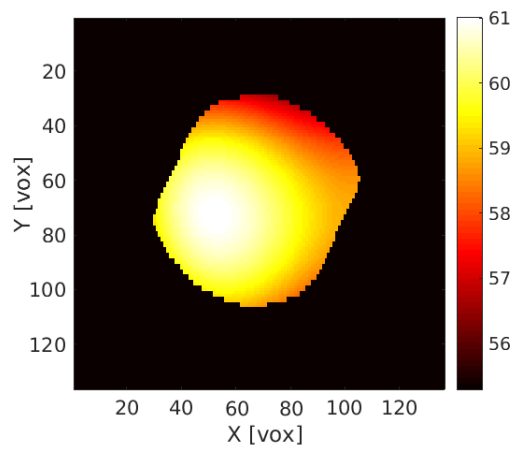

(b)

Fig. 9 Evolution of the front at different times of the scan acquisition, in voxels, reconstructed from the three modes for (a) $t=0$ and (b) 60 seconds

the tomography and the time-averaged interface as identified with the method presented in this paper. In this figure few steps are visible corresponding to jumps 
due to voxel rounding. The difference is always less than 1 voxel, corresponding to the $3 \mathrm{D}$ segmented uncertainty. The standard deviation of the difference is 0.36 voxel showing a good agreement in those two results. However the time evolution cannot be obtained with classical reconstruction procedures.

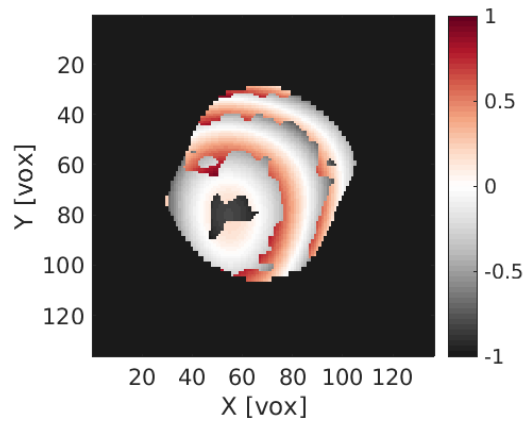

Fig. 10 Difference, in voxels, of the surface height between the mean identified surface with the proposed approach and the surface extracted from the segmented (voxelized) 3D volume.

\subsection{Case 2: during the entire test}

The above described identification procedure is now applied to identify the fluid front over the entire pressure-driven flow experiment. The analyzed test is composed of $42180^{\circ}$-scan series. To simulate the acquisition of a fast $4 \mathrm{D}$ procedure, only a single projection per invasion state has been selected from each scan in the series for a projection angle that evolves constantly with time just as if the experiment would have been conducted in 4 full turns of the sample (approximately 10 projections per turn). Projections at 3 times (7-21-40) and 3 different angles $\beta=\left[240^{\circ}, 0^{\circ}, 292^{\circ}\right]$ are shown figure 11 .

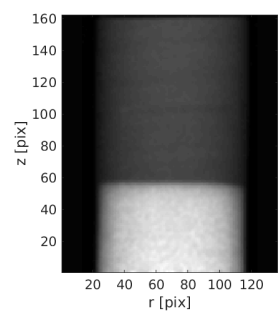

(a)

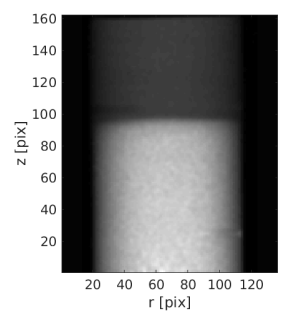

(b)

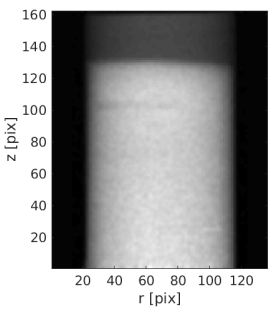

(c)

Fig. 11 Projections of the sample during the pressure-driven flow test (a) step 7, $\beta=240^{\circ}$, (b) step $21, \beta=0^{\circ}$ and (c) step $40, \beta=292^{\circ}$ 
The time regularization has been chosen as a 4 th order polynomial function because the front shape changes more rapidly during a full fluid advance as compared to a single scan. 5 time unknowns are identified per mode. The entire identification is composed of 5 independent modes, after which the residual does not decrease any further. The residual field after convergence is shown figure 12 . The residuals are low around the interface at the surface position, which validates the choice of surface regularizations.

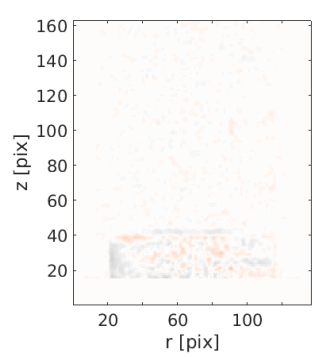

(a)

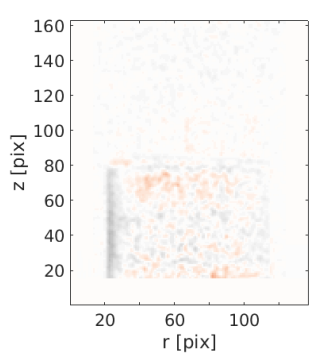

(b)

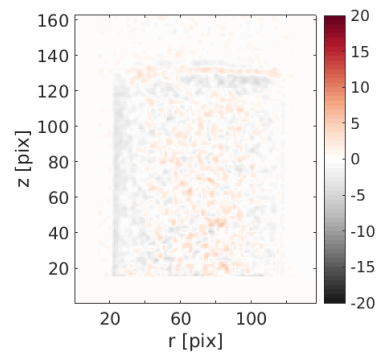

(c)

Fig. 12 Residual field at convergence of the procedure, with the correction of 5 identified modes during the test (a) step $7, \beta=240^{\circ}$, (b) step $21, \beta=0^{\circ}$ and (b) step $40, \beta=292^{\circ}$

The three first identified modes are shown figure 13 . The first mode with a very high amplitude, (from 20 to 120 ), is non linear in time and describes mostly the mean fluid invasion of the volume. The other modes, with smaller amplitudes, act as corrections to the first mode.

\section{Conclusion and discussion}

In order to follow a fast invasion process (water invading a porous sandstone), a new methodology has been introduced, based on a time regularization and a model reduction technique. Such a procedure allows to resolve high rate invasion (up to $5 \mathrm{~Hz}$ ) that cannot be captured using classical tomographic acquisitions and reconstructions. The method relies on a space and time regularization through a choice of a suited basis, and is based on a projection-based identification of the unknown parameters.

To further reduce the sought parameters, a Proper Generalized Decomposition has been designed, to capture "modes" one at a time until the residuals have been reduced down to an acceptable level. The residual field at the end of the procedure permits a validation of the chosen basis.

A first case example, addressed the question of a slow evolution during a $5 \mathrm{~Hz}$ scan. The residual field, after convergence of the identification procedure, does not reveal any salient unresolved features at the fluid front, which indicates that the front was well captured by the chosen description and the regularization is clearly well suited.

A second test case aimed at analyzing a fast pressure-driven flow experiment, and 40 radiographs acquired during about 4 full revolution of the sample were 


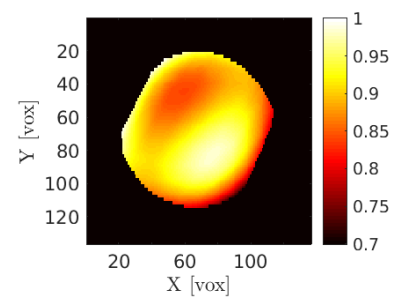

(a) $\sum_{i=1}^{N_{s}} f_{i}^{1} \varphi_{i}(x, y)$

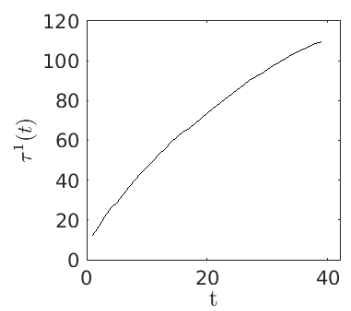

(d) $\sum_{j=1}^{N_{t}} g_{j}^{1} \theta_{j}(t)$

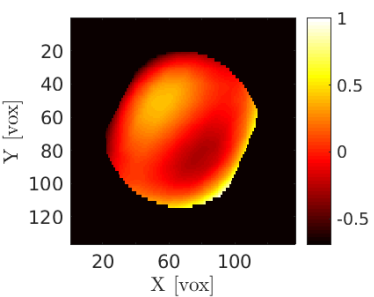

(b) $\sum_{i=1}^{N_{s}} f_{i}^{2} \varphi_{i}(x, y)$

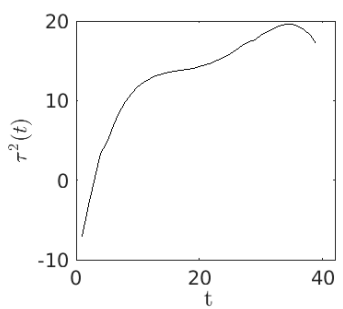

(e) $\sum_{j=1}^{N_{t}} g_{j}^{2} \theta_{j}(t)$

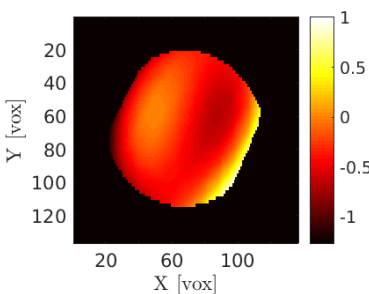

(c) $\sum_{i=1}^{N_{s}} f_{i}^{3} \varphi_{i}(x, y)$

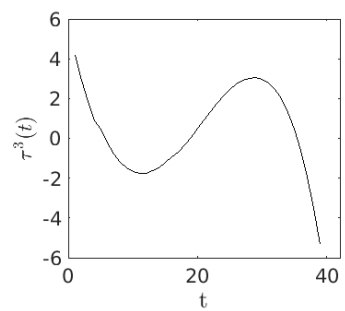

(f) $\sum_{j=1}^{N_{t}} g_{j}^{3} \theta_{j}(t)$

Fig. 13 Three first identified modes respectively (a-b-c) for the surface field and (d-e-f) for the time evolution

extracted from a longer time series. In contrast to the previous example, large displacements were observed and yet the proposed P-DVC algorithm could deal with such large changes.

The example applications of the method were performed at scale 4 (i.e., the volume was reduced in size by a factor of 4), so only small data volumes were considered. For the two test cases, because the surface was smooth, this resolution was enough to capture the fluid shape. The measurement at full resolution would be in the same spirit, with a higher computation time. With the actual procedure, each mode of case 1 is obtained in 55-65 minutes and each mode of case 2 in 4-6 minutes depending on the convergence rate of the fixed point procedure.

The simplicity/complexity of the problem (i.e., the number of degrees of freedom, in space and time) is conditioned by the scientific background of the experimentalist about the observed phenomenon. For example, the knowledge of fluid flow models composed of few parameters allows the problem to be easily solved with a small number of unknowns. In the opposite, no expectation about the experiment requires to leave much more freedom to the system (too many would lead to ill-conditioning). At convergence, the residual fields enable the quality of the parametrization and chosen model to be appreciated. It is then possible to enrich the studied basis (i.e., add an additional curvature of the front, space or time discontinuities, evolution of the front width etc. ).

The method could be applied to more complex cases such as imbibition or pressure-driven flow of water, or other fluids, into sample with cracks or shear 
bands. In such cases, the choice of the regularization basis would require particular attention (for example a finite element mesh may be suited, and a model based approach may also open a promising route).

\section{Acknowledgment}

Clément Jailin would like to especially thank the members of the Division of Solid Mechanics of Lund University for their warm welcome and for giving him the opportunity to work on this subject.

\section{References}

1. T. Bultreys, M. A. Boone, M. N. Boone, T. De Schryver, B. Masschaele, L. Van Hoorebeke, and V. Cnudde. Fast laboratory-based micro-computed tomography for pore-scale research: illustrative experiments and perspectives on the future. Advances in water resources, 95:341-351, 2016.

2. T. Bultreys, W. De Boever, and V. Cnudde. Imaging and image-based fluid transport modeling at the pore scale in geological materials: A practical introduction to the current state-of-the-art. Earth-Science Reviews, 155:93-128, 2016.

3. E Maire and PJ Withers. Quantitative X-ray tomography. International materials reviews, 59(1):1-43, 2014.

4. E Maire, C Le Bourlot, J Adrien, A Mortensen, and R Mokso. $20 \mathrm{~Hz}$ X-ray tomography during an in situ tensile test. International Journal of Fracture, 200(1-2):3-12, 2016.

5. K.J. Dobson, S. B. Coban, S. A. McDonald, J.N. Walsh, R. C. Atwood, and P. J. Withers. 4-d imaging of sub-second dynamics in pore-scale processes using real-time synchrotron x-ray tomography. Solid Earth, 7(4):1059, 2016.

6. S Akin, JM Schembre, SK Bhat, and AR Kovscek. Spontaneous imbibition characteristics of diatomite. Journal of Petroleum Science and Engineering, 25(3):149-165, 2000.

7. ER Rangel-German and AR Kovscek. Experimental and analytical study of multidimensional imbibition in fractured porous media. Journal of Petroleum Science and Engineering, 36(1):45-60, 2002.

8. S Youssef, H Deschamps, J Dautriat, E Rosenberg, R Oughanem, E Maire, and R Mokso. $4 \mathrm{D}$ imaging of fluid flow dynamics in natural porous media with ultra-fast X-ray microtomography. In International Symposium of the SCA, Napa Valley, California, volume $176,2013$.

9. C David, B Menéndez, and J-M Mengus. Influence of mechanical damage on fluid flow patterns investigated using CT scanning imaging and acoustic emissions techniques. Geophysical Research Letters, 35(16), 2008.

10. SA Hall, D Hughes, and S Rowe. Local characterisation of fluid flow in sandstone with localised deformation features through fast neutron imaging. In EPJ Web of Conferences, volume 6, page 22008. EDP Sciences, 2010.

11. V Cnudde, M Dierick, J Vlassenbroeck, B Masschaele, E Lehmann, P Jacobs, and L Van Hoorebeke. High-speed neutron radiography for monitoring the water absorption by capillarity in porous materials. Nuclear Instruments and Methods in Physics Research Section B: Beam Interactions with Materials and Atoms, 266(1):155-163, 2008.

12. C. Tötzke, N. Kardjilov, I. Manke, and S.E. Oswald. Capturing 3d water flow in rooted soil by ultra-fast neutron tomography. Scientific Reports, 7, 2017.

13. C-H Lee. Parametric study of factors affecting capillary imbibition in fractured porous media. PhD thesis, The Pennsylvania State University, 2011.

14. S Zou, F Hussain, J Arns, Z Guo, CH Arns, et al. Computation of Relative Permeability from In Situ Imaged Fluid Distributions at the Pore Scale. In International Petroleum Technology Conference. International Petroleum Technology Conference, 2016.

15. SA Hall. Characterization of fluid flow in a shear band in porous rock using neutron radiography. Geophysical Research Letters, 40(11):2613-2618, 2013.

16. Y Hajizadeh, E Amorim, M Costa Sousa, et al. Building trust in history matching: The role of multidimensional projection. In SPE Europec/EAGE Annual Conference. Society of Petroleum Engineers, 2012. 
17. B Basbug, ZT Karpyn, et al. Determination of relative permeability and capillary pressure curves using an automated history-matching approach. In SPE Eastern Regional/AAPG Eastern Section Joint Meeting. Society of Petroleum Engineers, 2008.

18. MR Deinert, J-Y Parlange, T Steenhuis, J Throop, K Ünlü, and KB Cady. Measurement of fluid contents and wetting front profiles by real-time neutron radiography. Journal of Hydrology, 290(3):192-201, 2004.

19. S. Gruener, Z. Sadjadi, H. E. Hermes, A. V. Kityk, K. Knorr, S. U. Egelhaaf, H. Rieger, and P. Huber. Anomalous front broadening during spontaneous imbibition in a matrix with elongated pores. Proceedings of the National Academy of Sciences, 109(26):10245-10250, 2012 .

20. S. Gruener, H.E. Hermes, B. Schillinger, S.U. Egelhaaf, and P. Huber. Capillary rise dynamics of liquid hydrocarbons in mesoporous silica as explored by gravimetry, optical and neutron imaging: Nano-rheology and determination of pore size distributions from the shape of imbibition fronts. Colloids and Surfaces A: Physicochemical and Engineering Aspects, 496:13-27, 2016.

21. R.T. Armstrong, H. Ott, A. Georgiadis, M. Rücker, A. Schwing, and S. Berg. Subsecond pore-scale displacement processes and relaxation dynamics in multiphase flow. Water resources research, 50(12):9162-9176, 2014.

22. F Hild, A Bouterf, L Chamoin, H Leclerc, F Mathieu, J Neggers, F Pled, Z Tomičević, and S Roux. Toward 4D mechanical correlation. Advanced Modeling and Simulation in Engineering Sciences, 3(1):17, 2016.

23. H Leclerc, S Roux, and F Hild. Projection savings in CT-based digital volume correlation. Experimental Mechanics, 55(1):275-287, 2015.

24. T Taillandier-Thomas, C Jailin, S Roux, and F Hild. Measurement of 3D displacement fields from few tomographic projections. In SPIE Photonics Europe, pages 98960L-98960L. International Society for Optics and Photonics, 2016.

25. M. H. Khalili, S. Brisard, M. Bornert, Aimedieu P., J.-M. Pereira, and J.-N. Roux. Discrete digital projections correlation: a reconstruction-free Method to quantify local kinematics in granular media by X-ray tomography. Experimental Mechanics, pages 1-12, 2017.

26. C Jailin, A Bouterf, M Poncelet, and S Roux. In situ $\mu$ CT-scan mechanical tests: Fast 4D mechanical identification. Experimental Mechanics, 2017.

27. A. Nouy. A priori model reduction through proper generalized decomposition for solving time-dependent partial differential equations. Computer Methods in Applied Mechanics and Engineering, 199(23):1603-1626, 2010.

28. P. Ladevèze. Nonlinear computational structural mechanics: new approaches and nonincremental methods of calculation. Springer Science \& Business Media, 2012.

29. J.C. Passieux and J.N. Périé. High resolution digital image correlation using proper generalized decomposition: PGD-DIC. International Journal for Numerical Methods in Engineering, 92(6):531-550, 2012.

30. LA Gomes Perini, J-C Passieux, and J-N Périé. A Multigrid PGD-based algorithm for Volumetric Displacement Fields Measurements. Strain, 50(4):355-367, 2014.

31. B. Schillinger and F. Grazzi. Artefacts in neutron ct - their effects and how to reduce some of them. Physics Procedia, 69:244 - 251, 2015.

32. GT Herman. Correction for beam hardening in computed tomography. Physics in medicine and biology, 24(1):81, 1979.

33. P Bésuelle, J Desrues, and S Raynaud. Experimental characterisation of the localisation phenomenon inside a Vosges sandstone in a triaxial cell. International Journal of Rock Mechanics and Mining Sciences, 37(8):1223-1237, 2000.

34. E Tudisco, Stephen A Hall, EM Charalampidou, N Kardjilov, A Hilger, and H Sone. Full-field measurements of strain localisation in sandstone by neutron tomography and 3D-volumetric digital image correlation. Physics Procedia, 69:509-515, 2015.

35. N Kardjilov, A Hilger, I Manke, M Strobl, M Dawson, S Williams, and J Banhart. Neutron tomography instrument CONRAD at HZB. Nuclear Instruments and Methods in Physics Research Section A: Accelerators, Spectrometers, Detectors and Associated Equipment, 651(1):47-52, 2011.

36. E. Tudisco, M. Etxegarai, S.A. Hall, E.M. Charalampidou, G. Couples, and N. Kardjilov. High-speed neutron tomography of fluid flow through rocks. in preparation, 2018.

37. W. Van Aarle, W.J. Palenstijn, J. De Beenhouwer, T. Altantzis, S. Bals, K.J. Batenburg, and J. Sijbers. The ASTRA Toolbox: A platform for advanced algorithm development in electron tomography. Ultramicroscopy, 157:35-47, 2015. 